\title{
Comparative Study Based on Two Kinds of Mobile Ad Hoc Network Routing Protocols
}

\author{
Yixin Yan $^{1 *}$ and Huamin Wang ${ }^{2}$ \\ ${ }^{1 * 2}$ School of Measurement and Communication, Harbin University of \\ Science \&Technology, Harbin, 150080, P. R. China \\ I*shiyu_wang@126.com, ${ }^{2}$ hmwzyx@163.com
}

\begin{abstract}
A mobile ad hoc network is a special kind of wireless mobile communication network, featuring equal status of all terminals, ad hoc, no need for prior arrangement of any network facilities or central control nodes, dynamically changing topology and bandwidth and energy restriction. In recent years, research on mobile ad hoc networks, especially research on media access control and routing protocols have research focus. In this paper, the well-known on-demand routing protocol AODV is analyzed and is improved as $A O D V-C L M P$, with a simulation platform used to evaluate its performance, with some meaningful conclusions drawn. In this paper, the origin, characteristics, application and current development of mobile ad hoc networks are introduced briefly, followed by research on two kinds of well-known on-demand routing protocols for mobile ad hoc networks, i.e. AODV and AODV-CLMP. Finally, commonly used network simulation software is compared to conduct evaluation and analysis of the performance of the AODV and AODV-CLMP protocols. Through the analysis of the simulation result of such evaluation indicators as average end-to-end delay, packet delivery fraction and throughput, the following conclusions are drawn: the average delay, packet deliveryfractionand throughput of $A O D V-C L M P$ protocol are superior to those ofAODV protocol, indicating thatAODV-CLMP protocol can transmit information more rapidly and steadily.
\end{abstract}

Keywords: mobile ad hoc network, routing protocol, MATLAB, AODV, AODV-CLMP

\section{Introduction}

A mobile ad hoc network is a communication network composed of wireless nodes or terminals autonomously, generally applied in situations requiring urgent or temporary creation of a network environment, featuring independence of fixed infrastructure, limited bandwidth and changeable topology. Network simulation is an important link in the communication network design and the key to analyzing network performance. Among numerous networking modes, due to the characteristics of no need for hardware infrastructure, multi-hop, no center, self-organization, etc., ad hoc networks have brought out their powerful advantages in the case of rapid establishment of temporary communications and requirement for distributed networks and become a hot spot in current network research. This paper involves research on ad hoc networks, with focus placed on the analysis of AODV and AODV-CLMP routing protocols [1]. Using MATLAB network simulation software, the excellence of AODV-CLMP routing protocol is highlighted. 


\section{AODV and AODV-CLMP Protocols}

\subsection{AODV Protocol}

2.1.1. Definition of AODV: AODV (Ad hoc on-demand distance vector routing) is a source-driven routing protocol. When a node needs to transmit information to the other nodes in the network, it must send RREQ (route request) message in the form of multicasting [2]. The RREQ message includes the network layer address of the initiating node and the destination node. When a neighboring node receives the RREQ, it will first judge whether the node itself is the destination node. If so, it will send RREP (route reply) to the initiating node; if not, it will search the table of nodes for a route to the destination node, and if any, it will send RREP to the source node in the form of unicasting, or it will transfer the RREQ for search.

\subsubsection{Characteristics of AODV:}

(1) AODV is a network layer protocol and an application layer protocol has to be triggered during route search each time, increasing the complexity of implementation.

(2) The frame size stipulated by IEEE 802.15 .4 is 127 bytes, of which at most 25 byte are used for MAC head and tail verification and 48 bytes are occupied by IPv6 head and UDP head, with only 54 bytes remaining free.

(3) In LR-WPAN, the topology is relatively simple, the network scale is relatively small and the position of nodes is not stable. In the design, consideration should be given to simplicity and energy conservation -related issues. These issues are not considered in the route framework and message design of AODV.

AODV (Ad hoc On-Demand Distance Vector Routing) protocol for mobile ad hoc networks is a routing protocol for route selection in mobile mesh networks and it can realize unicast and multicast routing [3]. This protocol is a typical protocol under the ondemand route generation mode in ad hoc networks.

2.1.3 AODV Realization Technology: AODV is a reactive routing protocol, which means that when a package is sent to the destination node, the source node starts the route lookup process in the network to find a corresponding route. On the contrary, many ordinary Internet protocols are proactive, which means that their route lookup doesn't rely on whether the nodes on the path will send packages, but that each node maintains a table of routes that contains information about routes reaching the other nodes. Routing information is exchanged periodically between the nodes to continuously update each node's own table of routes so that the network topology and changes can be reflected in a timely manner and consistent, timely and accurate routing information can be obtained. As is shown in the name of the protocol, AODV protocol is a distance vector routing protocol.

In AODV, the entire network is still unless there is demand for connection establishment, which means that only when a network node requires connection establishment, will a connection establishment request be broadcast. The other AODV nodes will transfer this request message and record the source node and the temporary route back to the source node. When a node that receives the connection request knows the route to reach the destination node, it will send this route information to the source node by taking the previously recorded temporary route back to the source node. Then the source node begins to use the route that goes through other nodes and has the minimum hops. In case of a broken link, route errors will be sent back to the source node, which will restart the route lookup process. 


\subsection{AODV Improvement-based AODV-CLMP Protocol}

2.2.1. Origin of AODV-CLMP: Cross-layer-based WMN multipath routing protocol (AODV-CLMP) proposed by us, an improved protocol based on AODV, is a routing protocol designed for the backbone network of WMN, with the main aim to make full use the resources in the network, especially free nodes and paths with wireless channels in good condition, so that network load can be more balanced to the greatest extent to improve network performance and meet the users' QOS requirements. Compared with traditional mobile ad hoc network routing that uses periodically sent detection packets to obtain network state information, the methods involves autonomous nodes and obtains the node resource use degree and channel competition state information through interaction with lower layers, greatly saving valuable network resources [4].

In a wireless network, there are usually two cases that will result in network congestion, with one being the reduced forwarding capacity of nodes due to insufficient node resources and the other one being the feature of shared wireless network channels. As each kind of business needs to compete for the right to use such channels, it results in severe channel competition in a certain area. Either of the above two cases may result in local network congestion, causing such phenomena as route lookup a number of times and packet loss, reducing the performance of a part of the network and even the entire network. In order to better solve such congestion problems, we include the two congestion perception-based cross-layer route selection parameters, i.e. node queue usage rate and frame successful delivery ration our new routing protocol.

Table 2-1. Cross-layer Routing Protocol (AODV-CLMP) Stack

\begin{tabular}{|c|c|}
\hline \multicolumn{2}{|c|}{ Higher Layer } \\
\hline Routing Layer & Routing Metrics Call Module \\
\hline \multirow{2}{*}{ MAC } & BOR Collection Module \\
& SFDR Collection Module \\
\hline \multicolumn{2}{|c|}{ Physical Layer } \\
\hline
\end{tabular}

Table 2-1 is a cross-layer protocol stack model for the new routing protocol. Due to the need to collect BOR and SFDR information, a parameter call module and a parameter collection module are added between the routing layer and the MAC layer. The parameter collection module is responsible for the statistics of the BOR and SFDR information of the node; the parameter call module provides interfaces for the routing layer to call the two kinds of routing metrics information. Each node maintains two tables of congestion state: BOR and SFDR.

2.2.2. AODV-CLMP routing discovery: When a certain node needs to obtain the route of another node, this node starts a route lookup process. The source node broadcasts a RREQ packet. Compared with the RREQ pack of AODV, this route has two additional path selection criteria: path minimum frame delivery success ratio and the first hop IP address [5].The minimum frame delivery success ratio of path represents the channel use situation of this RREQ packet in the area of the nodes its passes through, the larger the minimum frame successful delivery ratio, the better quality the path has. The RREQ packet structure is as shown in Figure 2-1. The discovery flow chart of AODV-CLMP routing is as shown in Figure 2-1. 


\section{Table 2-2. Route Discovery Flow Chart}

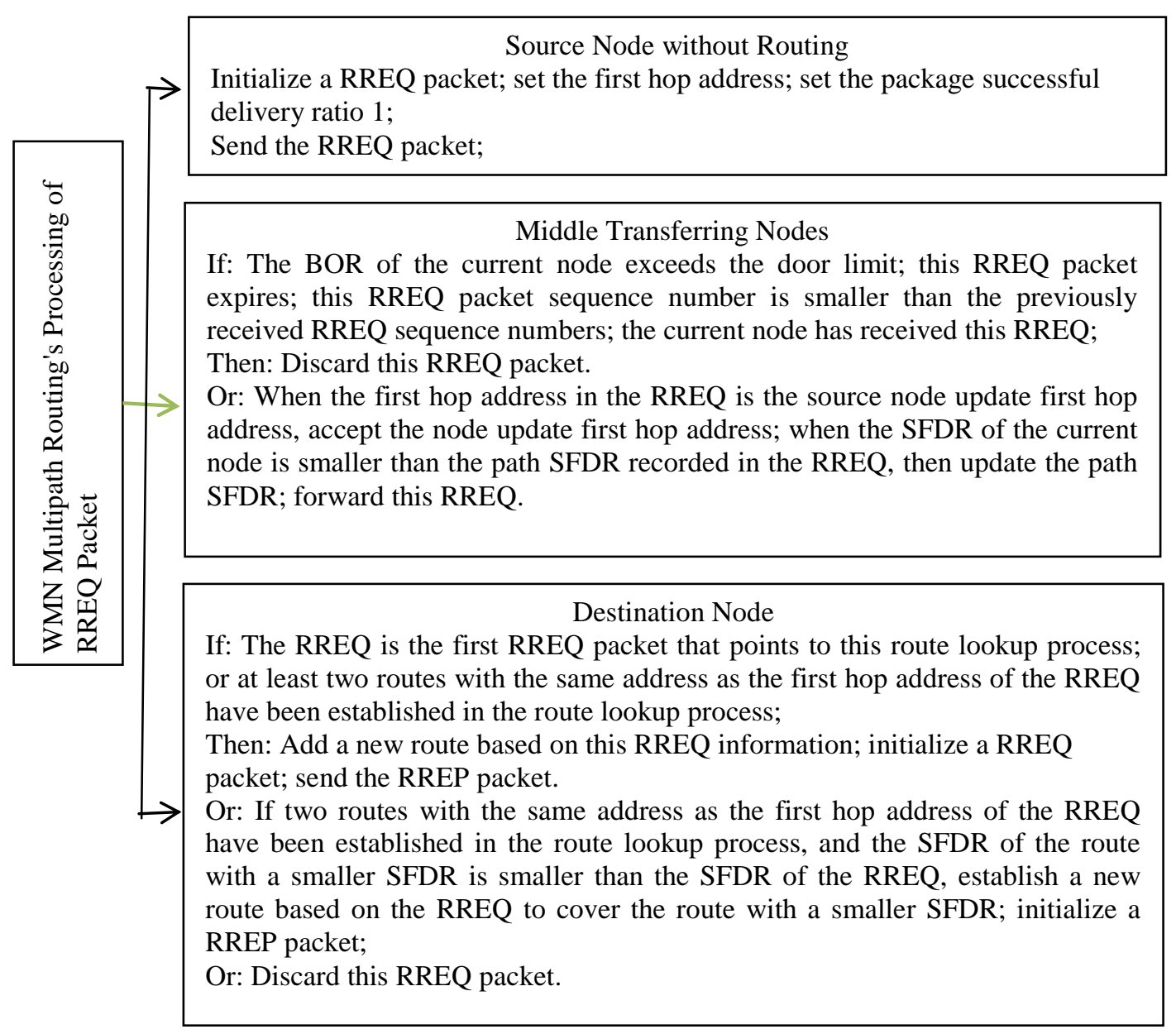

2.2.3. AODV-CLMP Routing Maintenance: The new route, like AODV, uses the mechanism that periodically sends information to neighboring nodes for maintenance. In case of a broken link from a certain node of a certain path to the node of the next hop, this node will send a RERR packet to the source node, and middle nodes can delete the routing information relating to the broken link after receiving the RERR. As the route is a multipath route, if the source node still has routes to the destination node, the source node will not start a new route lookup process. If all routes from the source node to the destination node fail, the source node will restart search for routes to the destination node.

2.2.4. AODV-CLMP Routing Business Assignment: After the source node broadcasts a RREQ packet, when the first RREP packet is received, the route that is found earliest will be used to send cached data packets. When the source node receives subsequent RREP packets, there will be a number of routes that can reach the destination node,then the transmission can be assigned to these routes. Use each packet assignment program. This program is used in the case of the presence of a number of routes to the destination node. 


\section{Simulation-based Research on the Performance of AODV and AODV-CLMP Protocols}

In Section 2.2, we give detailed description of the AODV-based improved AODVCLMP routing algorithm. The following is a simulation experiment on AODV-CLMP and AODV routing protocols based on different simulation scenes, with the simulation result analyzed. Our simulation scenes are mainly based on different network node densities.

\subsection{Simulation Environment Creation}

We use MATLAB as the simulation platform for the experiment, the network topology required in the experiment is generated by NSG tool and the whole experiment scene area is $2000 \mathrm{~m} \times 2000 \mathrm{~m}$, with 100 nodes randomly distributed, with simulation time of $100 \mathrm{~s}$. The Twe0RayoroundRefleetionMode is adopted for channel and wireless models, and the DCF(Distributed Coordination Function) of IEEE802.11 is used on the MAC layer. During the simulation, we will use two kinds of routing protocols to conduct UDP business performance analysis respectively. As the MAC layer has been optimized with the routing algorithm proposed by us, the optimized MAC and queue algorithm are used for AODV-CLMP routing protocol, while the default MAC and queue algorithm are used for the standard AODV routing protocol. The simulation experiment focuses on the simulation analysis of the UDP business performance of WMN in terms of network node density. The focus of the performance assessment is placed on the following aspects: (1) end-to-end data throughput (2) average end-to-end delay; (3) rate of the data packets successfully sent; (4) ratio of control messagesto data messages.

\subsection{Effect of Network Node Density on Routing Performance}

In MATLAB simulation, routing performance evaluation is conducted based on the random scenes with the number of nodes being 20,30, 40, 50, 60, 70, 80, 90 and 100 respectively, mainly involving the following aspects(l) end-to-end data throughput (2) average end-to-end delay; (3) rate of the data packets successfully sent; (4) ratio of control messages to data messages.

Figure 3-1 shows the simulation result of average network end-to-end delay under different network node densities. Overall, under four node densities, the time delay performance of AODV-CLMP is superior to that of AODV. First, AODV-CLMP chooses the nodes with a low queue occupancy rate to forward data packets through BOR parameters; second, AODV-CLMP avoids hot spot area involving fierce competition through SFDR parameters, and at the same time, the use of multipath transmission greatly reduces the waiting time of data packets in the queue, contributing to time delay performance of AODV-CLMP that is far superior to that of AODV. Within an appropriate scope, when the number of nodes increases, the opportunity of AODV-CLMP routing protocol finding a number of paths with good performance can be greatly increased, and even the time delay in data transmission can be reduced [6]. 


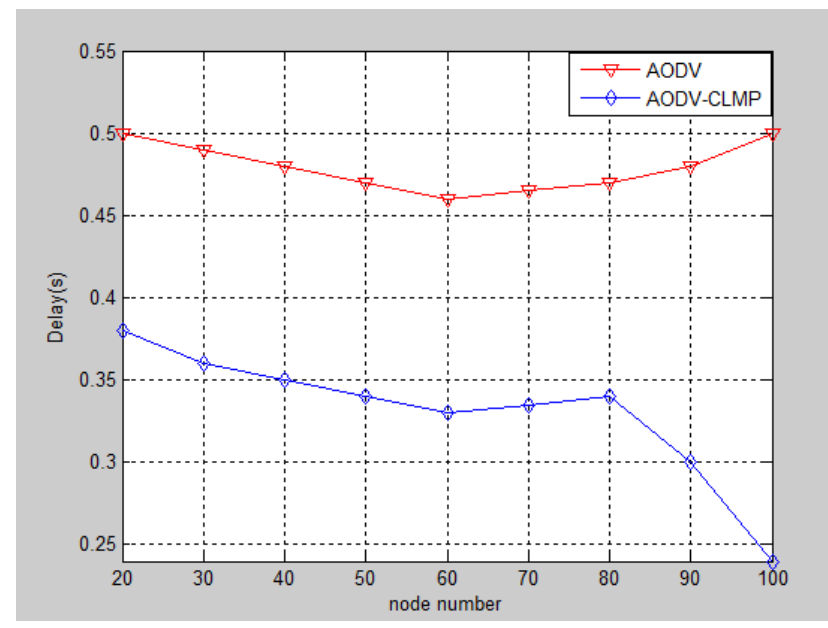

Figure 3-1. Number of Network Nodes and Time Delay

Figure3-2and Figure 3-3 respectively reflect the changes of the ratio of control packets to data packets, packet delivery success rate and network throughput with the network node density. As these three performance evaluation indicators are not independent of each other (for example, the higher the packet successful delivery rate, the higher the network throughput), we will discuss them together here.

The ratio of control packets to data packets reflects routing efficiency. Each time a data packet is sent successfully, the number of control packets that need to be sent gets smaller, indicating the high efficiency of routing protocols. As can be seen in Figure 3-2, under the same node density, the routing efficiency of AODV-CLMP is far superior to that of AODV. In AODV-CLMP, consideration is given to node congestion during the route lookup period and all selected nodes have a lot of resources available. Seen from the simulation scene setting, we injected six UDP flows into the network when 10 seconds passed, and these six UDP flows would initiatively avoid the paths selected by the six UDP flows operating in the network and use the nodes with small BOR; at the same time, we optimized the control packet forwarding strategies, and reduced the number of routing control packets in the network. The above measures have greatly reduced the routing control packets of AODV-CLMP and improved routing efficiency, path reliability and packet delivery rate, as shown in Figure 3-3.

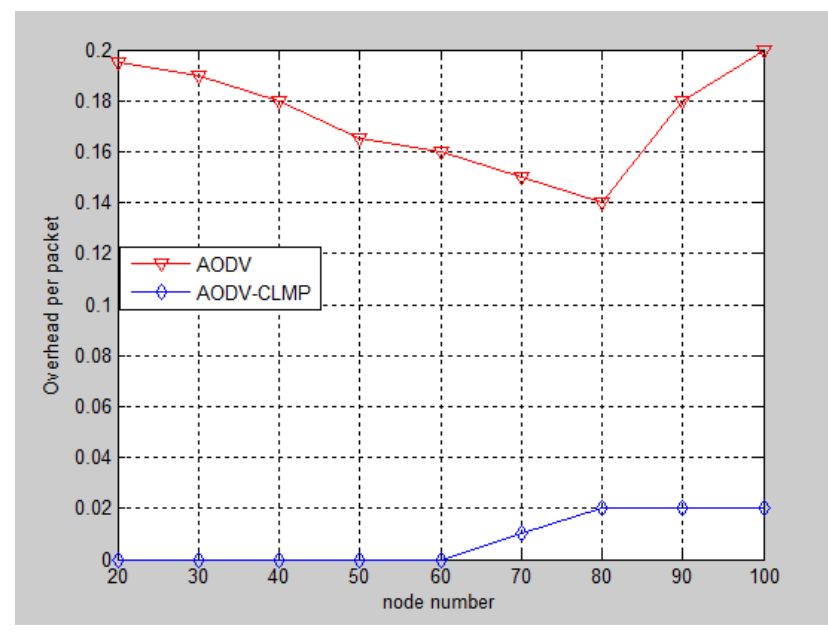

Figure 3-2. Number of Network Nodes and Ratio of Control Packets and Data Packets 


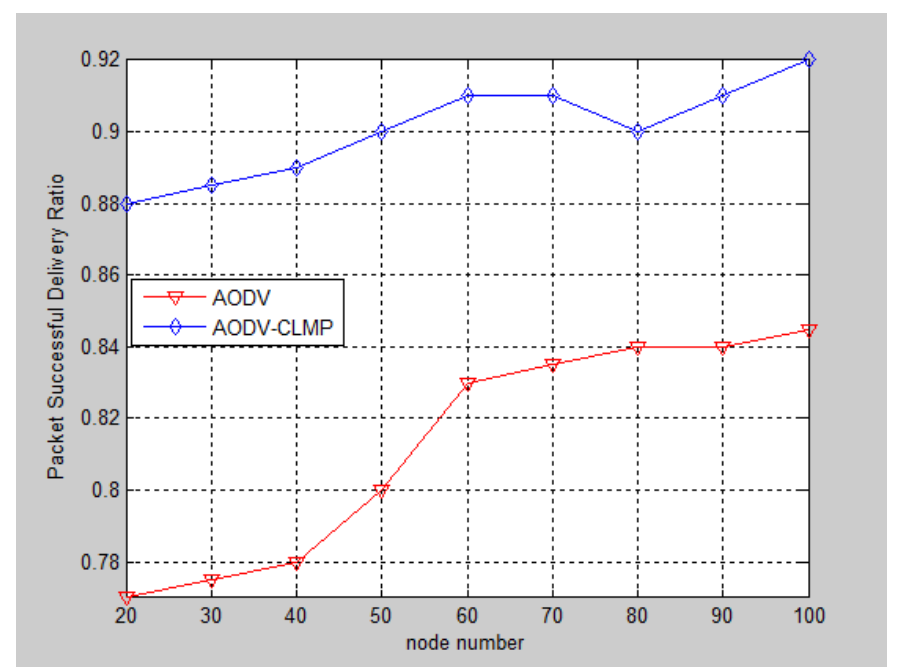

Figure 3-3. Number of Network Nodes v Packet Successful Delivery Ratio

It can be seen in Figure 3-2 that as for AODV-CLMP, in the case of a relatively small number of network nodes, the ratio of control packets to data packets is very low. Then with the increase in the network node density, the ratio gradually rises, which is because that the increase in the network nodes has contributed to the broadcasting of control packets within a wider scope and increased the number of control packets. AODV-CLMP is an improved multipath routing protocol. When the source node is able to find a number of paths, it adopts multipath transmission; if the source node has only one path, it adopts single-path transmission [7]. The increase in the number of nodes can greatly increase the opportunities of the routing protocol fining a number of paths with good performance. Multipath routing can not only balance network load, which is fully reflected in Figure 32 , but also enhance routing reliability, thereby increasing the packet successful delivery rate[8], which is fully reflected in Figure 3-3. Figure3-4 shows the simulation result of routing throughput performance under different node densities. Coupled with Figure 3-3, due to the high packet successful delivery ratio of AODV-CLMP, accordingly, its throughput is also superior to that of AODV.

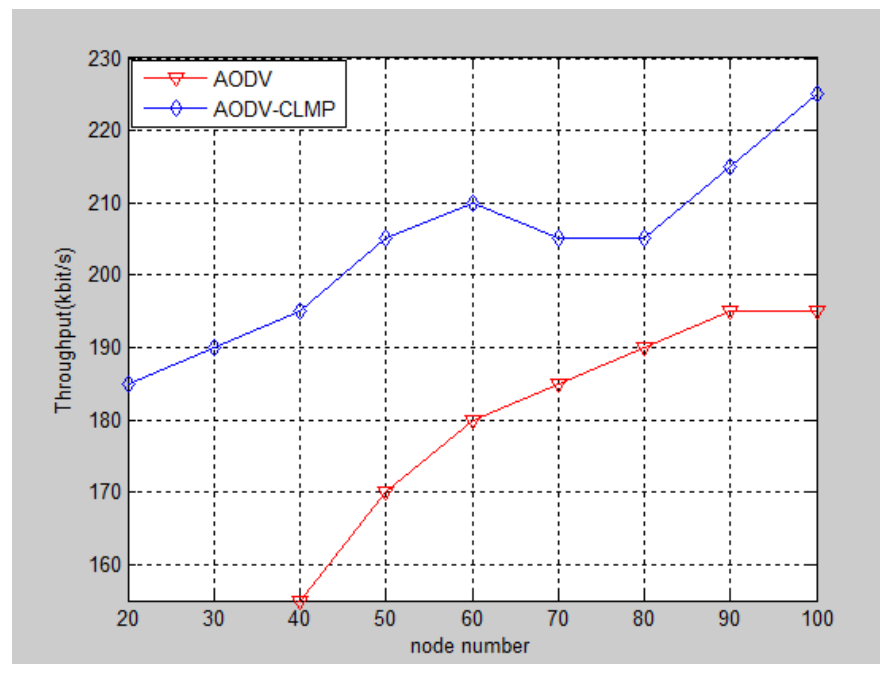

Figure 3-4. Number of Network Nodes v Throughput 


\section{Conclusions}

In this paper, the origin, characteristics, application and current development of mobile ad hoc networks are introduced briefly, followed by research on two kinds of well-known on-demand routing protocols for mobile ad hoc networks, i.e. AODV and AODV-CLMP. Then MATLAB is used to complete the simulation experiments on AODV-CLMP and AODV as routing protocols based on different simulation scenes, with simulation conducted based on different network node densities, with the simulation result showing that all properties of AODV-CLMP routing protocol is superior to those of AODV protocol, which proves the excellence of an AODV-CLMP routing algorithm improved based on AODV.

\section{Acknowledgements}

This study was financed partially by Higher Education Key Laboratory for Measuring and Control Technology and Instrumentation of Heilongjiang Province, Harbin University of Science and Technology.

\section{References}

[1] L. Haiyan, L. Daoquan, W. Huaicai and C. Qiguang, "Comparative Study Based on Two Kinds of Mobile Ad Hoc Network Routing Protocols", Internet Security, vol. 3, no. 37, (2009), pp. 14-17.

[2] L. Lan-ying and L. Chang-dong, "An Improved Algorithm of LEACH Routing Protocol in Wireless Sensor Networks", Harbin University of Science and Technology, vol. 2, (2015), pp. 75-79.

[3] W. Jingrong, "Study on the MAC Layer and Relevant Technologies of Mobile Ad Hoc Network", Beijing University of Posts and Telecommunications, vol. 6, (2013), pp. 78-83.

[4] Y. Bengong, L. Guilan and X. Shaobin, "GloMoSim-based Simulation Study on Mobile Ad Hoc Network Routing Protocols”, Computer Science and Development, vol. 12, no. 12, (2013), pp. 105-108.

[5] X. Luyang, "AODV-based Improved Cross-layer Multipath WMN Routing Protocol”, South China University of Technology, vol. 6, (2010), pp. 165-166.

[6] Y. Tongjun, "Study on Military Mobile Ad Hoc Network Routing Design", Graduate School of National University of Defense Technology, vol. 10, (2012), pp. 14-21.

[7] Y. Shaowen, "NS2-based Study on Mobile Ad Hoc Network Routing Protocols", Harbin University of Science and Technology, vol. 3, (2007), pp. 56-57.

[8] M. Xiaofeng, L. Zhihui and H. Liang, "SUMO-based Routing Protocol Simulation Study", Computer Engineering, vol. 1, no. 37, (2011), pp. 107-110. 